\title{
Comparison of Trichothecene Biosynthetic Gene Expression between Fusarium graminearum and Fusarium asiaticum
}

\author{
Theresa Lee ${ }^{1 \dagger *}$, Seung-Ho Lee ${ }^{2 \dagger}$, Jean Young Shin ${ }^{1}$, Hee-Kyoung Kim³ ${ }^{3}$, Sung-Hwan Yun ${ }^{3 *}$, Hwang-Yong Kim ${ }^{1}$, \\ Soohyung Lee ${ }^{1}$ and Jae-Gee Ryu ${ }^{1}$ \\ ${ }^{1}$ Microbial Safety Team, National Academy of Agricultural Science, Rural Development Administration (RDA), Suwon 441- \\ 707, Korea \\ ${ }^{2}$ Ginseng Research Division, National Institute of Horticultural \& Herbal Science, RDA, Eumseong 369-873, Korea \\ ${ }^{3}$ Department of Medical Biotechnology, Soonchunhyang University, Asan 336-745, Korea
}

(Received on November 2, 2013; Revised on November 25, 2013; Accepted on November 25, 2013)

Nivalenol (NIV) and deoxynivalenol (DON) are predominant Fusarium-producing mycotoxins found in grains, which are mainly produced by Fusarium asiaticum and $F$. graminearum. NIV is found in most of cereals grown in Korea, but the genetic basis for NIV production by $F$. asiaticum has not been extensively explored. In this study, 12 genes belonging to the trichothecene biosynthetic gene cluster were compared at the transcriptional level between two NIV-producing $F$. asiaticum and four DONproducing $F$. graminearum strains. Chemical analysis revealed that time-course toxin production patterns over 14 days did not differ between NIV and DON strains, excluding $F$. asiaticum R308, which was a low NIV producer. Both quantitative real-time polymerase chain reaction and Northern analysis revealed that the majority of TRI gene transcripts peaked at day 2 in both NIV and DON producers, which is $\mathbf{2}$ days earlier than trichothecene accumulation in liquid medium. Comparison of the gene expression profiles identified an NIV-specific pattern in two transcription factor-encoding TRI genes (TRI6 and TRI10) and TRI101, which showed two gene expression peaks during both the early and late incubation periods. In addition, the amount of trichothecenes produced by both DON and NIV producers were correlated with the expression levels of TRI genes, regardless of the trichothecene chemotypes. Therefore, the reduced production of NIV by R308 compared to NIV or DON by the other

\footnotetext{
These authors contributed equally to this work.

*Co-corresponding authors.

T. Lee

Phone) +82-31-290-0451, FAX) +82-31-290-0407

E-mail) tessyl1@korea.kr

S-H. Yun

Phone) +82-41-530-1288, FAX) +82-41-530-3085

E-mail)sy14@sch.ac.kr
}

strains may be attributable to the significantly lower expression levels of the TRI genes, which showed early expression patterns.

Keywords : deoxynivalenol, Fusarium graminearum, $F$. asiaticum, nivalenol, TRI gene expression

Fusarium head blight (FHB), or scab, of small grains (such as barley and wheat) is a serious disease that reduces crop productivity and can result in mycotoxin contamination. When infected, harvested grains may contain trichothecene mycotoxin such as deoxynivalenol (DON) or nivalenol (NIV), as well as their acetyl derivatives (and/or zearalenone), which are commonly found in grains worldwide (Gale et al., 2011; Pasquali et al., 2010; Zhang et al., 2007).

A major causal agent of FHB is the member of the Fusarium graminearum species complex (FGSC), which contains over 15 phylogenetically distinct species (also known as lineages), including $F$. graminearum and $F$. asiaticum (Mule et al., 1997; O'Donnell et al., 2000; Wang et al., 2011). Among these species, F. graminearum is ubiquitously distributed around the world (Desjardins, 2006; Yli-Mattila, 2011), while $F$. asiaticum is frequently found in Asia, including Korea and Japan (Karugia et al., 2009; Lee et al., 2009; Lee et al., 2011).

Fusarium graminearum and $F$. asiaticum share morphological, cultural, and molecular characteristics, and even toxicology. Generally, F. graminearum strains produce DON and its acetyl derivatives (3-ADON or 15-ADON), while $F$. asiaticum strains produce NIV and its acetyl derivative (4-ANIV). Both mycotoxins belong to tricyclic sesquiterpenes and share the type B trichothecene backbone, which is mostly synthesized by enzymes and regulators encoded by the genes (TRI) located at the trichothecene 
biosynthetic gene cluster (Desjardins, 2006). Differences in chemical structure between DON and NIV (i.e., presence or absence of hydroxyl group at the $\mathrm{C} 4$ position) originated from nucleotide variations in the two TRI genes (TRI13 and TRI7) between two chemotype strains (Lee et al., 2001; Lee et al., 2002). NIV is known to be more toxic than DON (Eriksen et al., 2004) and to exert a synergistic effect when mixed with DON (Sobrova et al., 2010). In addition, the quantity of each trichothecene differs between DON and NIV strains; the relative amount of DON produced by a DON-producing FGSC isolate (typically $F$. graminearum) is higher than that of NIV by a NIV-producing $F$. asiaticum isolate. These species are normally simultaneously present in host crops in Korea. Due to the more frequent occurrence and resulting economic loss, DON is better characterized than NIV.

At this time, extensive comparisons between NIV and DON strains have been performed for genes controlling trichothecene production and sexual reproduction, such as those located at the mating type (MAT) loci (Kim et al., 2012) and trichothecene gene clusters (Kimura et al., 2003; Lee et al., 2001). These studies have revealed more than $80 \%$ nucleotide similarity between the two chemotype strains. As described above, nucleotide polymorphisms of TRII3 and TRI7 are known to determine trichothecene chemotypes of $F$. graminearum and $F$. asiaticum (Lee et al., 2001; Lee et al., 2002). Alterations in individual MAT gene expression is thought to be due to variations in selffertility between two chemotype strains; the majority of NIV-producing $F$. asiaticum showed a lower self-fertility than the highly fertile DON-producing $F$. graminearum strains (Kim et al., 2012; Lee et al., 2009, 2012). At this time, genome sequencing of a Korean $F$. asiaticum strain is almost complete, which will allow comparisons to be made at the whole genome level (Yun et al., unpublished data). However, the quantitative difference in trichothecene production by two chemotype strains has not been explored. Therefore, we compared the expression of members of the TRI gene clusters in DON- and NIV-producing strains to determine whether differences in the TRI gene expression level contribute to the differential ability of the two chemotype strains to produce trichothecenes.

\section{Materials and Methods}

Fungal strains and nucleic acid preparation. Six strains were used for comparison. Among the four DON-producing F. graminearum strains, PH-1 (Trail and Common, 2000) and H-11 (Lee et al., 2001) were 15 acetyl DON (15ADON) producers, while H7-4 and H7-11 were 3 acetyl
DON (3-ADON) producers. PH-1 was isolated from wheat in the United States, H-11 from corn, and H7-4 and H7-11 from barley grown in Korea. Fusarium asiaticum strains SCK04 (Kim et al., 2005) and R308, which were isolated from Korean barley and rice, respectively, produce NIV and 4 acetyl NIV (4-ANIV) simultaneously. All Korean strains are available upon request. For trichothecene production, fungal strains were grown in a defined liquid medium ( $1 \mathrm{~g}, \mathrm{KH}_{2} \mathrm{PO}_{4}, 0.5 \mathrm{~g} \mathrm{MgSO}_{4} \cdot 7 \mathrm{H}_{2} \mathrm{O}, 0.5 \mathrm{~g} \mathrm{KCl}, 1.14$ $\mathrm{g}$ agmatine sulfate, $30 \mathrm{~g}$ sucrose, $0.2 \mathrm{ml}$ trace elements, and distilled water to $1 \mathrm{~L}$; Gardiner et al., 2009; Leslie and Summerell, 2006) for 14 days, and mycelia were harvested every 2 days. All cultures were replicated three times. Mycelia were used for nucleic acid extraction, and culture liquid was saved for toxin analysis. Fungal genomic DNA was extracted as described previously (Leslie and Summerell, 2006). Total RNA was extracted from the mycelial mat using the easy-spin ${ }^{\mathrm{TM}}$ total RNA Extraction Kit (iNtRON Biotechnology, Daejeon, Korea) according to the manufacturer's protocol. Total RNA $(5 \mu \mathrm{g})$ was used to synthesize cDNA using the SuperScript III First-Strand Synthesis System for reverse transcription-polymerase chain reaction (RT-PCR; Invitrogen, Carlsbad, CA, USA).

Quantitative real-time PCR. Twelve TRI genes were analyzed (Table 1). To determine the expression level of each gene, a set of gene-specific PCR primers spanning the coding regions of each gene was designed using the Primer3 Program (http://bioinfo.ut.ee/primer3-0.4.0/; Table $1)$. As an endogenous gene, translation elongation factor 1 alpha $(E F 1 \alpha)$ was selected among several housekeeping genes as a control (Kim and Yun, 2011). Quantitative real-time PCR (qPCR) reactions were performed in an iCycler iQ5 ${ }^{\text {TM }}$ Real Time PCR Detection System (Bio-Rad, Hercules, CA, USA). The PCR thermal cycling conditions were as follows: $95^{\circ} \mathrm{C}$ for $3 \mathrm{~min}$, followed by 45 cycles at $95^{\circ} \mathrm{C}$ for $10 \mathrm{~s}, 60^{\circ} \mathrm{C}$ for $20 \mathrm{~s}, 72^{\circ} \mathrm{C}$ for $20 \mathrm{~s}$, and finally $95^{\circ} \mathrm{C}$ for $10 \mathrm{~s}$ and $65^{\circ} \mathrm{C}$ for $5 \mathrm{~s}$. The PCR reaction contained 10 $\mu 1$ of $2 \times$ iQ SYBR ${ }^{\circledR}$ Green Supermix (Bio-Rad), $0.5 \mu$ of each primer $(10 \mathrm{pM}), 1 \mu \mathrm{l}$ of template cDNA (12.5 ng), and sterile bi-distilled water to a final volume of $20 \mu$. Quantification values were automatically determined using the Bio-Rad CFX Manager version 1.6, and the threshold cycle $(\mathrm{Ct})$ values were determined. The standard curve represents a plot of the $\mathrm{Ct}$ versus log DNA concentration. In all experiments, appropriate negative controls containing no template were subjected to the same procedure to exclude or detect DNA contamination. Each sample was amplified in duplicate with three repeats in every experiment, and final $\mathrm{Ct}$ values are presented as an average. Relative levels 
Table 1. Genes analyzed and primers used in the study

\begin{tabular}{|c|c|c|}
\hline \multirow{2}{*}{$\begin{array}{l}\text { Gene } \\
\text { (Ref) }\end{array}$} & \multirow{2}{*}{ Function } & Forward primer $\left(5^{\prime} \rightarrow 3^{\prime}\right)$ \\
\hline & & Reverse primer $\left(5^{\prime} \rightarrow 3^{\prime}\right)$ \\
\hline \multirow{2}{*}{$\begin{array}{l}\text { FPP } \\
\text { (Desjardins et al., 2006) }\end{array}$} & \multirow{2}{*}{ Farnesyl phyrophosphate synthase } & TTTGGCAAGCCCGAACACATT \\
\hline & & GCGGATCTGGCCAACAACCTTCT \\
\hline \multirow{2}{*}{$\begin{array}{l}\text { TRI3 } \\
\text { (Kimura et al., 2007) }\end{array}$} & \multirow{2}{*}{ Trichothecene 15-O-acetyltransferase } & CTTGCAGGGATATCAAGAAATGTTACGA \\
\hline & & CTCGCCTGTTGTAGTTCGCTTGATTT \\
\hline \multirow{2}{*}{$\begin{array}{l}\text { TRI4 } \\
\text { (Kimura et al., 2007) }\end{array}$} & \multirow{2}{*}{ Trichodiene oxygenase } & TCGAGGCACAACAGAAGGGTATCC \\
\hline & & AATGTCGGCCTTGGTGGTGTC \\
\hline \multirow{2}{*}{$\begin{array}{l}\text { TRI5 } \\
\text { (Desjardins et al., 2006) }\end{array}$} & \multirow{2}{*}{ Trichodiene synthase } & CCAGGAAACCCTACACTCGTCTAAG \\
\hline & & TGGCCGCCTGCTCAAAGAAC \\
\hline \multirow{2}{*}{$\begin{array}{l}\text { TRI6 } \\
\text { (Kimura et al., 2007) }\end{array}$} & \multirow{2}{*}{ Transcription factor } & GGCATTACCGGCAACACTTCAA \\
\hline & & CATGTTATCCACCCTGCTAAAGACC \\
\hline \multirow{2}{*}{$\begin{array}{l}\text { TRI8 } \\
\text { (Kimura et al., 2007) }\end{array}$} & \multirow{2}{*}{ Trichothecene 3-O-esterase } & GCTACTTTGGACTCAATTCG \\
\hline & & CATACTGTACYGCAAGTTCTG \\
\hline \multirow{2}{*}{$\begin{array}{l}\text { TRI9 } \\
\text { (Kimura et al., 2007) }\end{array}$} & \multirow{2}{*}{ Unknown } & AGCCGCTAAACTGATCGACTCATA \\
\hline & & GCTTTGGCTGCGACCCATAT \\
\hline \multirow{2}{*}{$\begin{array}{l}\text { TRI10 } \\
\text { (Brown et al., 2001) }\end{array}$} & \multirow{2}{*}{ Regulatory gene } & GTGGCCGGGACGCTTCAAT \\
\hline & & ATCCGTCAAGTCTTCCCATCTCAT \\
\hline \multirow{2}{*}{$\begin{array}{l}\text { TRI11 } \\
\text { (Kimura et al., 2007) }\end{array}$} & \multirow{2}{*}{ Isotrichodermin 15-oxygenase } & AAGTACTTCACCCGACCAAACGAC \\
\hline & & CGGCAAGGCGAATGTCAAAC \\
\hline \multirow{2}{*}{$\begin{array}{l}\text { TRI12 } \\
\text { (Kimura et al., 2007) }\end{array}$} & \multirow{2}{*}{ Major facilitator superfamily transporter } & TCCACAGTCATCTTTCCCCAGTCT \\
\hline & & CTCCCAGTGCCATAGCGAAGTAGT \\
\hline \multirow{2}{*}{$\begin{array}{l}\text { TRI14 } \\
\text { (Dyer et al., 2005) }\end{array}$} & \multirow{2}{*}{ Unknown } & CTGGGAACCTACGCATCAAACATT \\
\hline & & CGAATGAGCTGCCCAATGATGT \\
\hline \multirow{2}{*}{$\begin{array}{l}\text { TRI101 } \\
\text { (Kimura et al., 2007) }\end{array}$} & \multirow{2}{*}{ Trichothecene 3-O-acetyltransferase } & GTGGGACTCTGGGATTACGACTTT \\
\hline & & GTCCACTCCTTATCCGCCTTCAA \\
\hline
\end{tabular}

of the genes were calculated by comparing the $\mathrm{Ct}$ values using EF1 $\alpha$ as the endogenous reference and assigning the gene expression level on day 2 in PH-1 (FPP, TRI4, TRI5, TRI6, TRI10, TRI11, TRII2, and TRI101) and H-11 (TRI3, TRI8, TRI9, and TRI14) as 1 (Table 3).

Northern analysis. Gene expression was also analyzed using Northern analysis. To prepare probes, each TRI gene was amplified with the qPCR primers noted above and purified using a QIAquick Gel Extraction Kit (Qiagen, Hilden, Germany). Northern analysis was performed using standard methods (Sambrook and Russell, 2001).

Toxin analysis. Each agmatine-amended liquid culture was filtered through filter paper (No. 1; Whatman, Maidstone, Kent, UK), and $500 \mu \mathrm{l}$ of the filtrate was directly used in mixture preparation with acetonitrile:water (1:1) solution for liquid chromatography/mass spectrometry (LC/MS) analysis (Hewlett-Packard, Palo Alto, CA, USA). The culture samples were screened for the production of DON, NIV, and their acetyl derivatives (15-ADON, 3-ADON, and 4-ANIV), as described previously (Lee et al., 2011).

Statistical analysis. To analyze changes over time in gene expression by each strain, a general linear model (GLM) was used for the one-way analysis of variance (ANOVA). When the null hypothesis was rejected $(P<0.05)$, means were compared using Duncan's multiple range tests. The 
same procedure was also applied to compare the amount of toxin produced by all tested strains at one specific time point. All statistical analysis was performed using SAS Enterprise Guide 4.3 (SAS Institute, Cary, NC, USA).

\section{Results}

Toxin accumulation by strains during culture. For all fungal strains examined (excluding H7-4), both DON and NIV production were initially detected at days 4 or 6 , and their accumulation gradually peaked at day 12 or 14 (Fig. 1). In contrast, production patterns of their acetyl derivatives (3-ADON, 15-ADON, or 4-ANIV) differed from the nonacetyl forms as the toxin induction and the peak level was observed at least 2 days earlier; the toxin levels continuously decreased after peaking (Fig. 1). However, the patterns observed in strains PH-1, H7-4, and H7-11 were slightly biased as second peaks occurred at day 8. Quantities of total trichothecenes (DON + acetyl forms) produced in three $F$. graminearum strains ( $\mathrm{PH}-1, \mathrm{H} 7-4$, and $\mathrm{H} 7-11$ ) did not fluctuate significantly between the fungal strains, ranging from 13.5 to $29.0 \mu \mathrm{g} \mathrm{ml}^{-1}$; H-11 produced more trichothecenes ( 1.7-3.5-fold higher) than the other three strains. One NIV-producing $F$. asiaticum SCKO4 strain also produced a similar level of trichothecenes (NIV + 4-ANIV), while the other $F$. asiaticum $\mathrm{R} 308$ produced significantly lower (5.6-20.0-fold) amounts of trichothecene than DON producers and SCKO4 (Fig. 1).

Comparison of gene expression. Relative expression of a specific gene determined by qPCR among the strains was statistically compared (Table 2), and the results supported the patterns shown in Fig. 2. Based on both qPCR and Northern hybridization data, transcript accumulations of the TRI genes could be categorized into at least four patterns. First, six TRI genes (TRI4, TRI5, TRI6, TRI10, TRI11, and TRI12) showed an early induction in most strains (excluding $F$. asiaticum R308, which was a poor NIV producer and showed very low basal expression levels throughout the entire incubation time). qPCR analysis showed that the gene expression peaked at day 2, abruptly decreased at day 4, and stayed low until day 14 (Fig. 2). Northern data clearly supported this type of gene expression in TRI4 and TRI5 (Fig. 3). Based on the qPCR data, F. graminearum PH-1 showed a slightly different pattern from the other strains for five genes (TRI5, TRI6, TRI10, TRI11, and TRI12), in which the transcript accumulation peaked at day 4 instead of day 2. However, Northern hybridization analysis supported

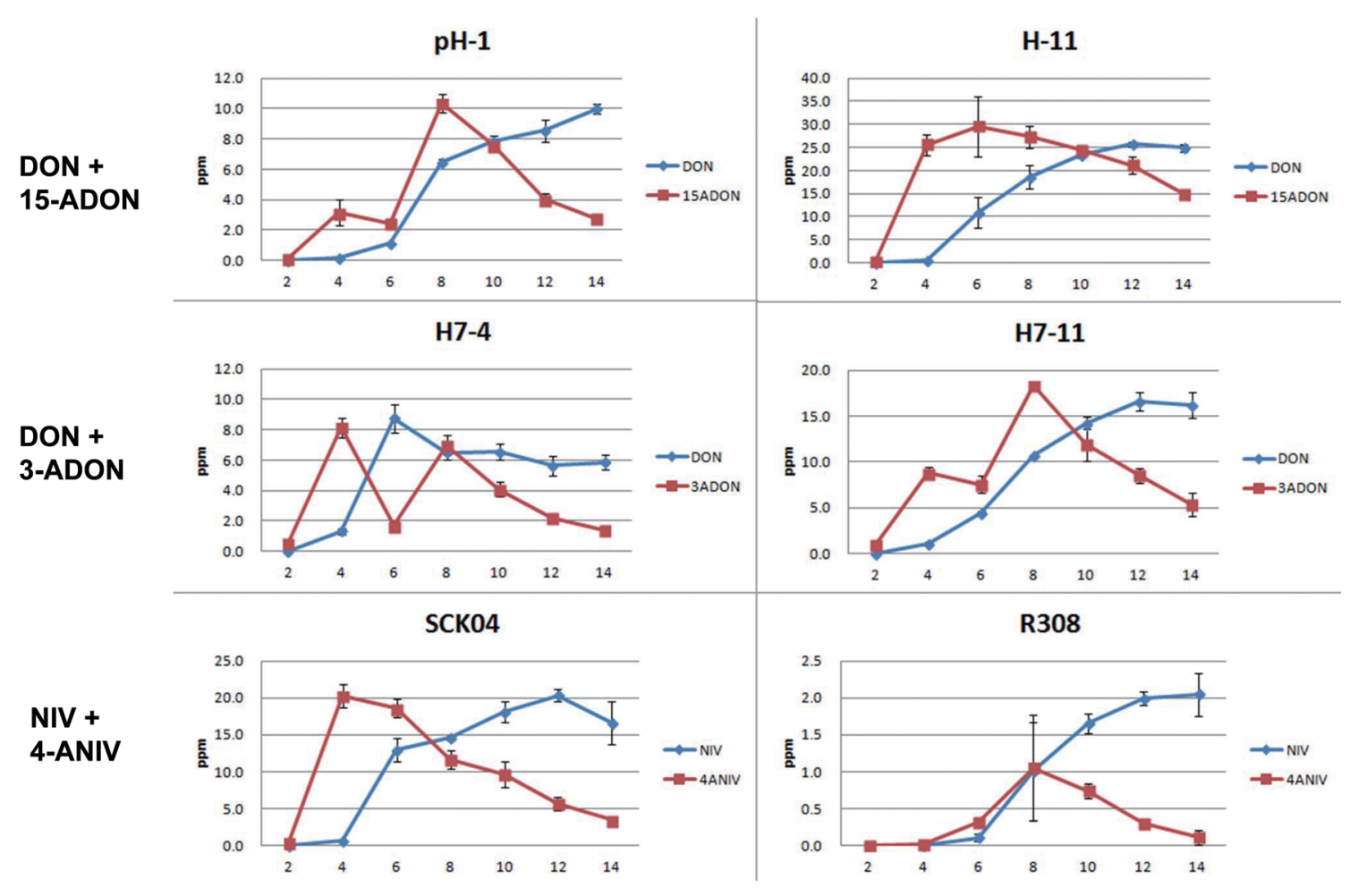

Fig. 1. Toxin accumulation by DON and NIV strains in agmatine-amended liquid medium. Vertical bars indicate the standard deviation of the observed values at the time point. 


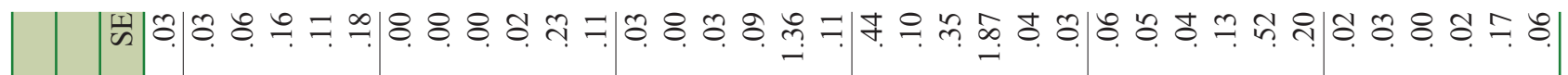

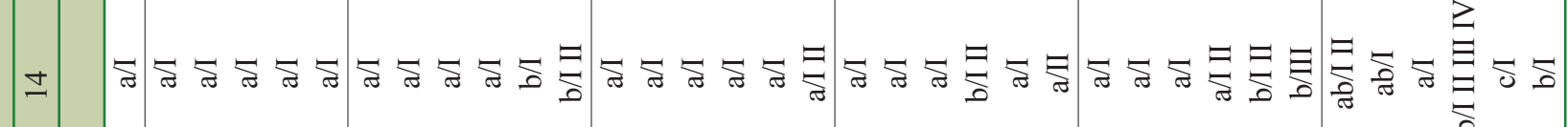

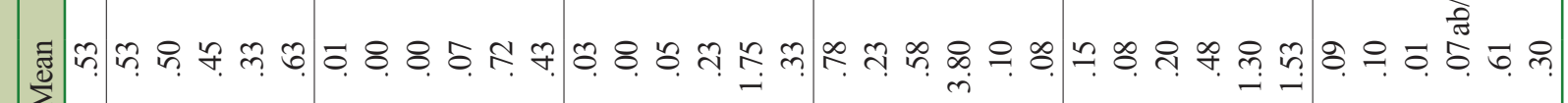

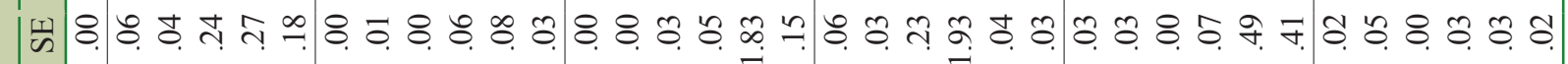

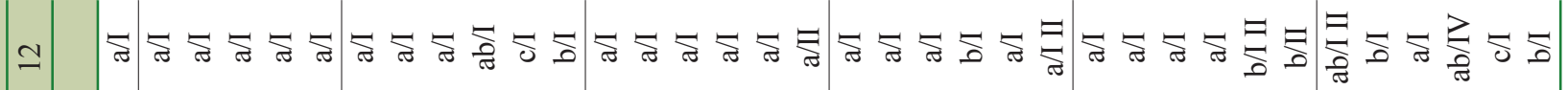
₹

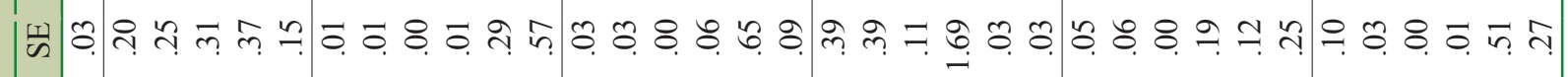

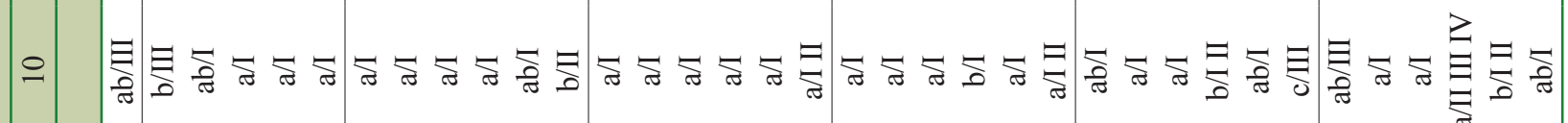

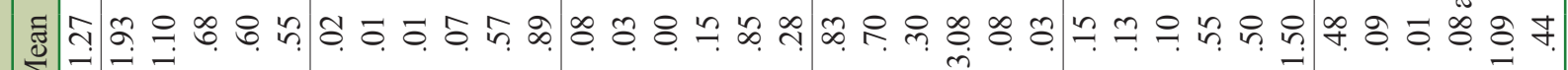

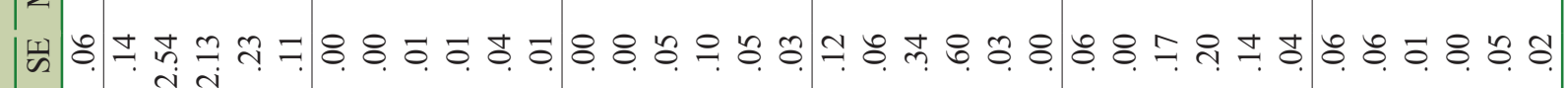

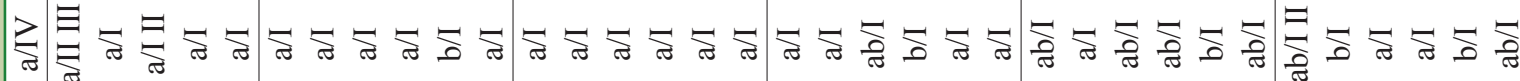
馬

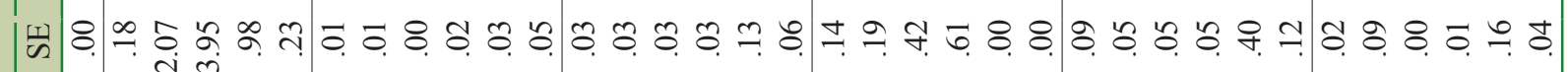

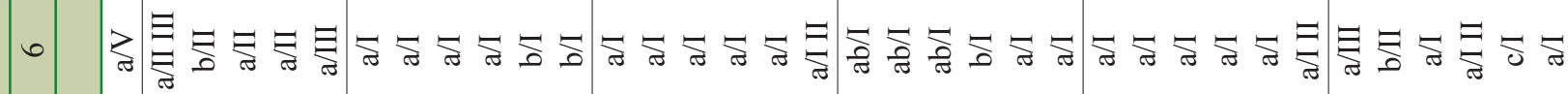

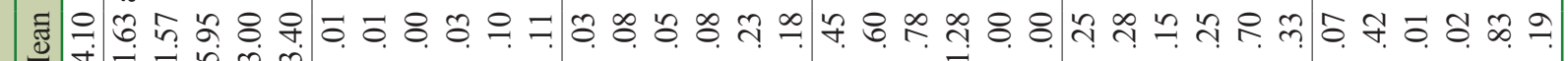

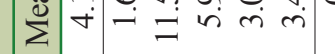

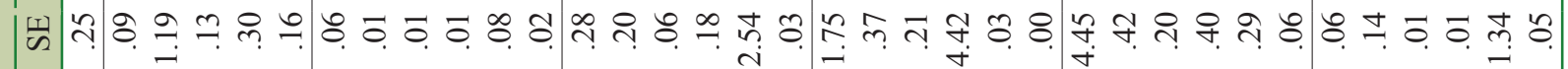

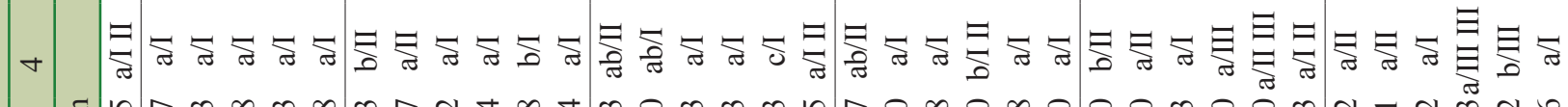

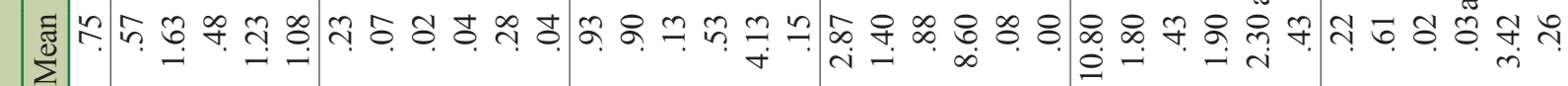

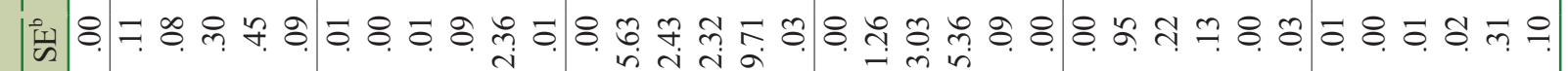

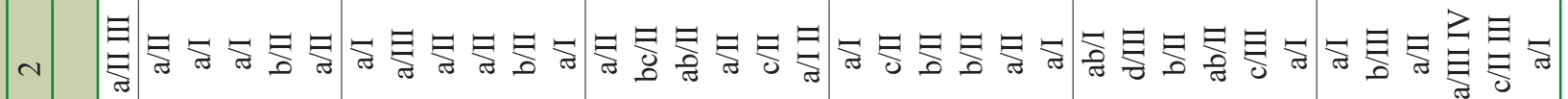

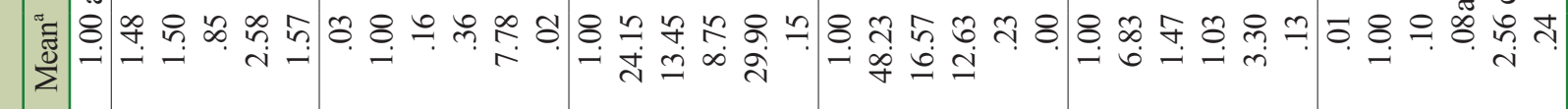

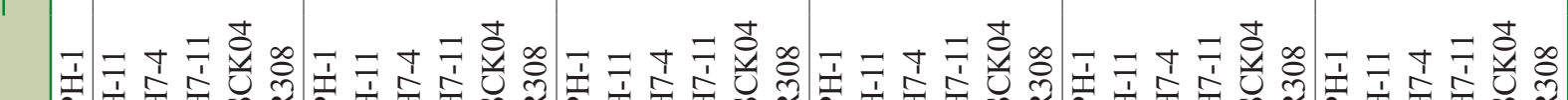

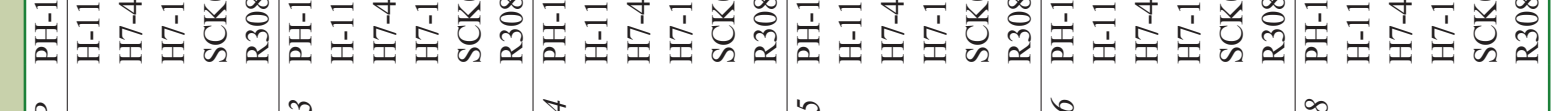
位 产 芯 椨 胥

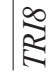




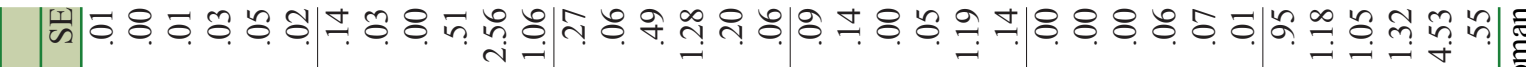

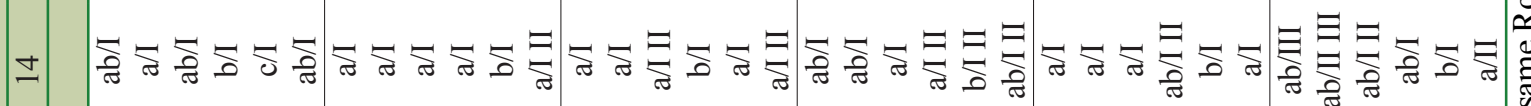

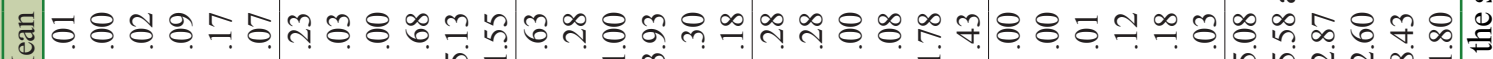

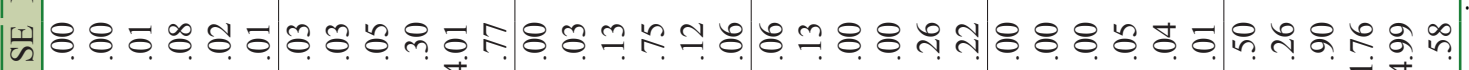

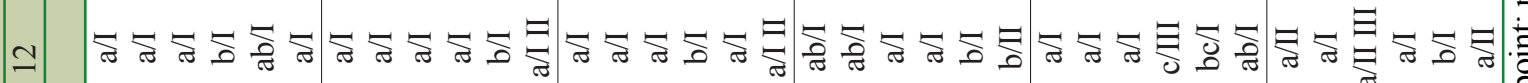

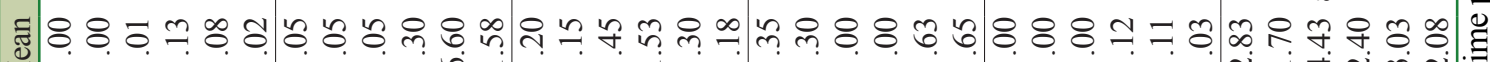
$\frac{10}{4}$ 请

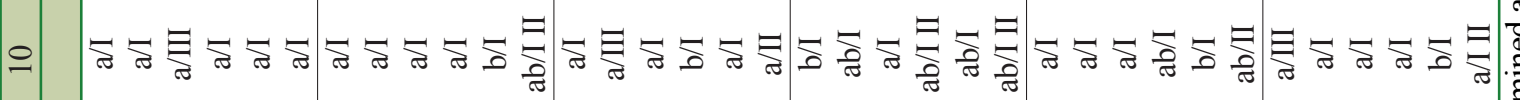

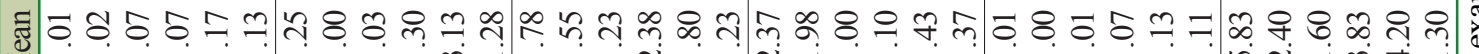
ล

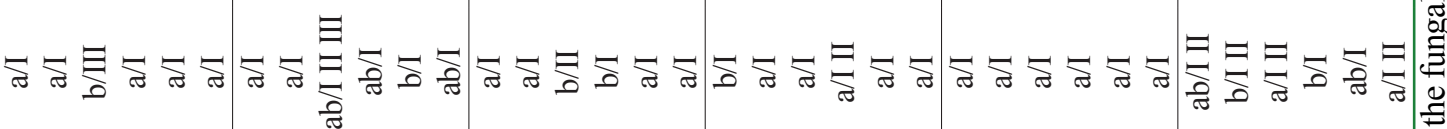

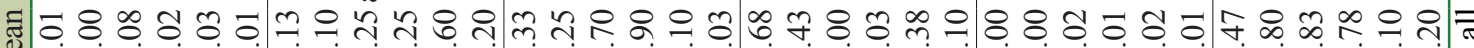
$\sum$ क्ष

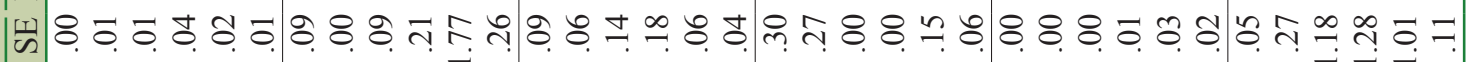

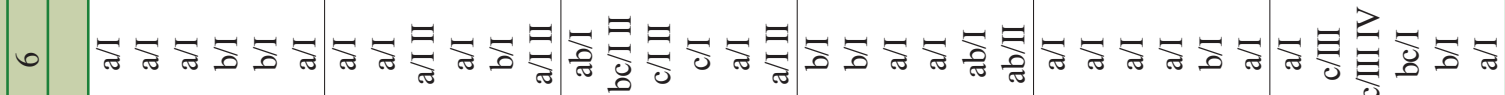

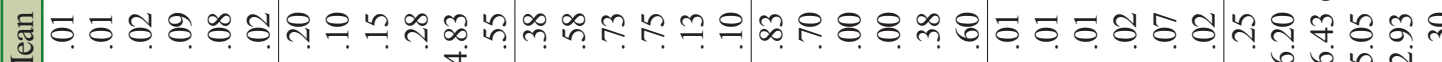

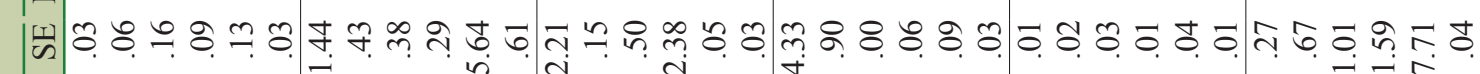
竞

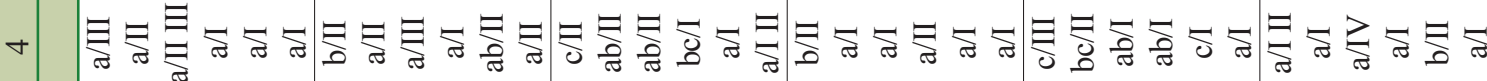
$\neg$ 구 구 㠃

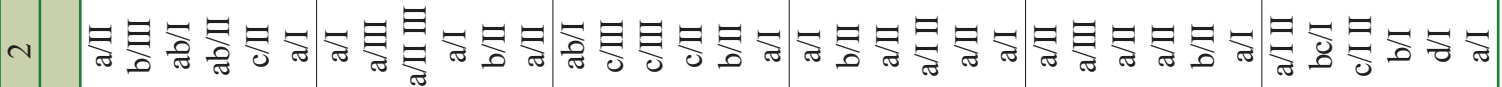

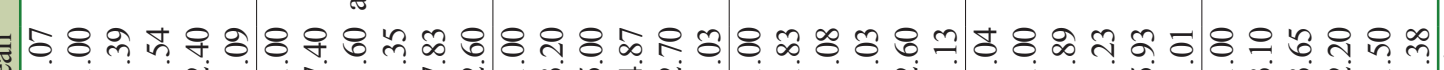



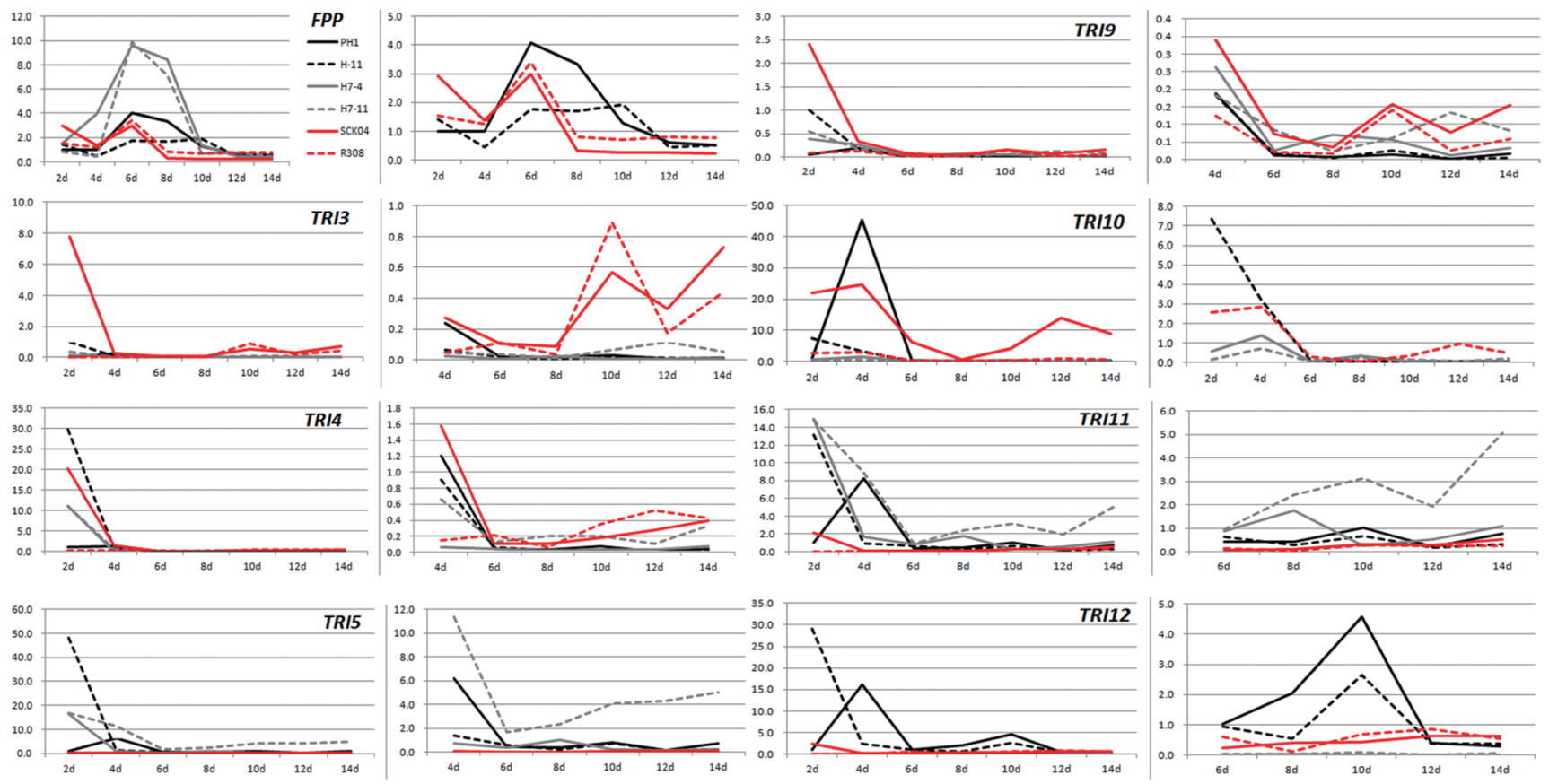

3
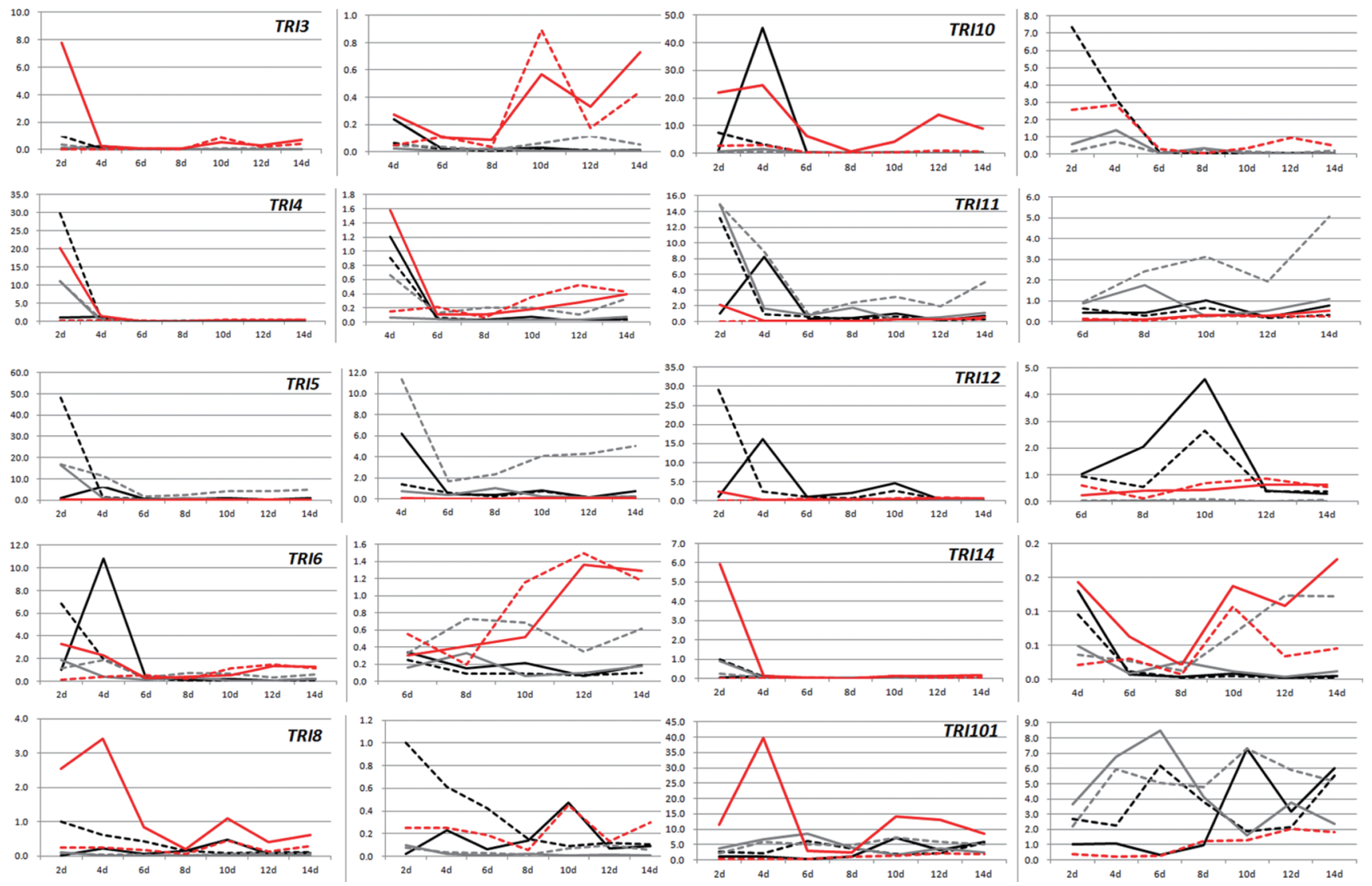

Fig. 2. Transcript accumulation of individual TRI genes in agmatine-amended liquid medium based on qPCR. The $x$-axis represents days (d) after inoculation for trichothecene production in liquid medium. The $y$-axis represents relative amounts of transcripts. Data for each gene are shown in two separate graphs; left in each set show the entire graph, and an enlarged graph for the indistinct data is shown on the right. The positions and nucleotide sequences of the primers used in qPCR are shown in Table 1. Data shown are the mean values obtained from three independent biological samples, and detailed statistical information is shown in Table 2.

that the gene expression of TRI4 and TRI5 in PH-1 were consistent with other strains in this group. Despite the earlier expression, several fungal strains showed variations at later points. Unlike the DON-producing $F$. graminearum strains, both NIV-producing $F$. asiaticum strains SCKO4 and R308 maintained significant expression levels of TRI6 and TRIIO during the relatively late incubation periods, showing two expression peaks at days 2 or 4 and 12 or 14 (Fig. 2, Table 2). In addition, the TRI12 transcript reached the second peak on day 10 in 15-ADON-producing $F$. graminearum strains (H-11 and $\mathrm{PH}-1)$. Second, the FPP gene showed a middle induction period in all strains, but a slight difference was noted between $F$. graminearum and $F$. asiaticum. In $F$. graminearum, the gene expression was significantly detected on day 2, peaked at days 6 or 8 , and gradually decreased until day 14 , while the expression levels significantly decreased after day 6 in $F$. asiaticum. The $F$. asiaticum-specific expression pattern was also demonstrated based on Northern hybridization. Third, TRI101 could be grouped as a relatively constitutive expression type in all strains, excluding $F$. asiaticum R308, which showed a constant expression during the late incubation periods (days 8-14), although the expression levels were relatively low. Fourth, the remaining TRI genes (TRI3, TRI8, TRI9, and TRI14) showed no significant level of transcript accumulation during the entire incubation period in any strain, excluding $F$. asiaticum SCKO4. Notably, these TRI genes were expressed with the early induction pattern 


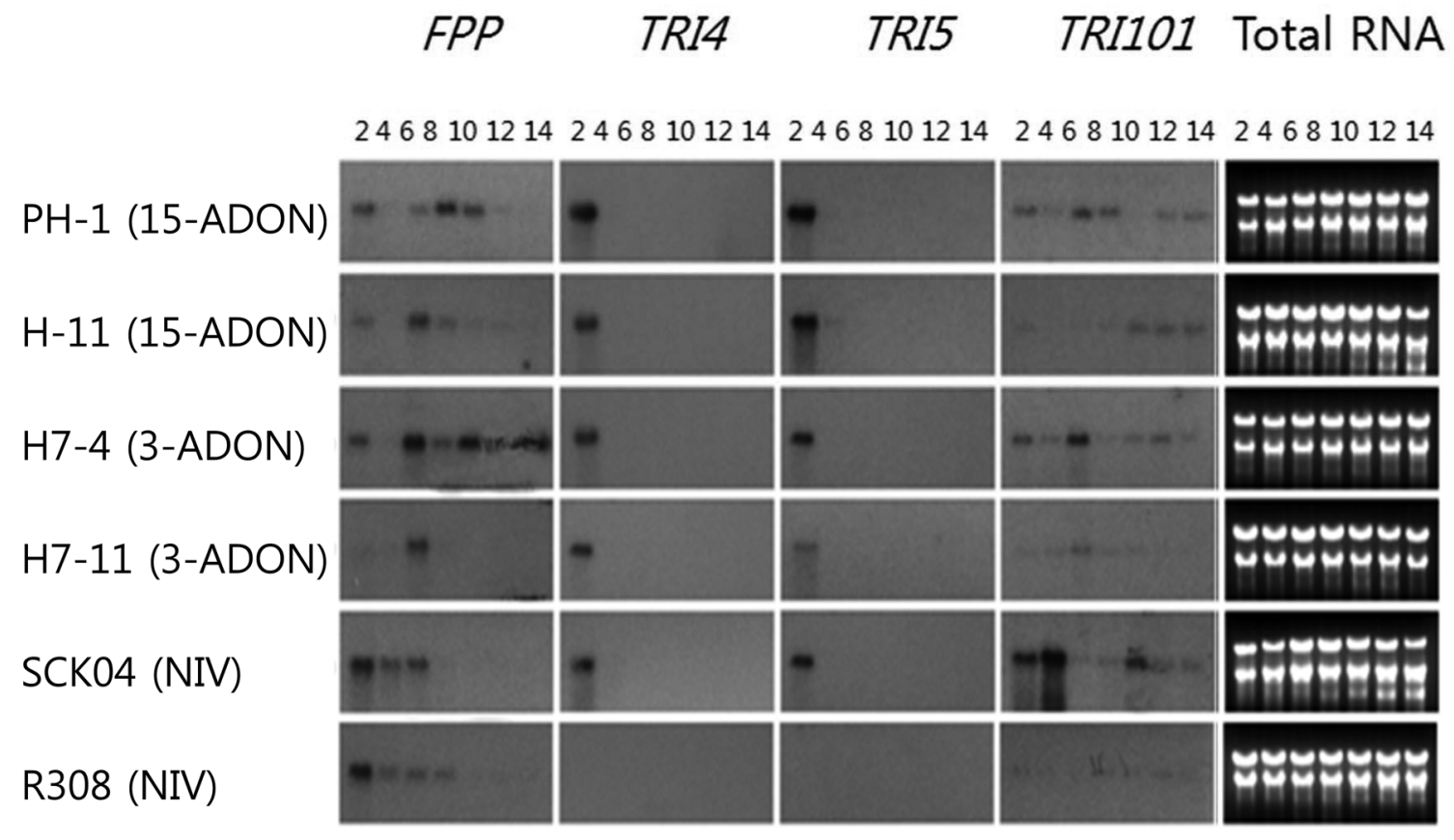

Fig. 3. Northern blots of F. graminearum and F. asiaticum strains probed with four genes located the trichothecene gene cluster. Incubation times (days after inoculation) are indicated above the gels.

in only SCKO4. TRI7 and TRI13 were excluded from the comparison because they were directly involved in NIV synthesis and defective in DON production.

\section{Discussion}

The trichothecene accumulation pattern revealed that acetyl derivatives (15-ADON, 3-ADON, or 4-ANIV) were detected first, followed by non-acetylated forms (DON or NIV). Acetylated trichothecenes peaked at day 4, while the synthesis of non-acetylated forms continued increasing. This pattern was expected since acetyl derivatives are known to form before the non-acetyl forms (Desjardins, 2006). Timing of toxin production was also similar to the previous analyses in barley (Boddu et al., 2005), wheat (Hallen-Adams et al., 2011), and liquid culture (Doohan et al., 1999; Jiao et al., 2008; Merhej et al., 2010). Thus, no difference occurred in the induction timing of trichothecene production between DON and NIV in any strains examined. Gene expression profiling revealed that the expression of most TRI genes were induced 2 days earlier when compared to time-course toxin accumulation, indicating that high levels of TRI transcript accumulation is a prerequisite for initiating the biosynthetic pathway of DON or NIV. In this regard, the early induction patterns of six TRI genes (TRI4, TRI5, TRI6, TRI10, TRII1, and $T R I 12)$ in the high trichothecene producers, and the other four genes (TRI3, TRI8, TRI9, and TRI14) in only SCKO4, strongly support that two transcriptional regulators encoded by TRI6 and TRIIO positively regulated the expression of these TRI genes, leading to the synthesis of metabolic enzymes and other proteins required for trichothecene production in both $F$. graminearum and $F$. asiaticum. Considering the strong transcript signals of TRI3 and TRI8 based on Northern analysis of $F$. sporotrichioides (Peplow et al., 2003), the possibility exists that the lack of detectable gene expression in the second set of TRI genes (TRI3, TRI8, TRI9, and TRI14) in the DON-producing F. graminearum could have been due to experimental artifacts. Based on this information, their expression patterns would be the early induction type, similar to SCKO4.

The availability of detailed gene expression profiles among different trichothecene chemotypes allowed us to identify the possible chemotype-specific gene expression patterns. The most significant difference between DON and NIV producers was observed in the transcription regulatorencoding TRI genes (TRI6 and TRI10): the maintenance of gene expression during the late incubation periods (i.e., days 10-14) in both NIV producers (SCKO4 and R308), as well as the early induction in SCKO4 (TRIO) or SCKO4 and R308 (TRI10). Similarly, TRI101 encoding trichothecene 3-O-acetyltransferase was expressed during the same late periods in only two NIV producers. In contrast, the expression levels of FPP-encoding farnesyl pyrophosphate 
synthetase were restricted during the early incubation times (days 2-6) in both NIV producers, while the gene expression was maintained until day 10 in DON producers. These variations were specific to NIV-producing $F$. asiaticum strains since they were mostly observed in two NIV producers regardless of the quantities of NIV they produced. Moreover, SCKO4 is a relatively high trichothecene producer. However, further investigations are required to confirm that these differences are associated with genetic diversity between DON- and NIV-producing species of the FGSC. Excluding these cases, no significant difference was observed at the transcriptional level in the early-induced genes between three DON-producing $F$. graminearum strains, and one NIV-producing $F$. asiaticum $\mathrm{SCKO} 4$ strain that produced trichothecenes at similar levels as the DON producers. This indicated that the amount of trichothecene accumulated in agmatine-amended liquid medium is proportional to the expression level of TRI genes, regardless of the DON/ NIV chemotypes. In this regard, the observation that the amount of NIV produced by $F$. asiaticum is lower than that of DON by $F$. graminearum may be due to the relatively lower levels of TRI gene expression in F. asiaticum than $F$. graminearum. To confirm this hypothesis, more NIVproducing $F$. asiaticum should be investigated for TRI gene expression under various culture conditions, including solid cereal plants. Additionally, exploring the regulatory networks is important, including signal transduction pathways controlling the biosynthetic pathway for trichothecene production, to explore the genetic elements responsible for the difference in trichothecene quantities between the two chemotypes. In addition to the NIV-specific gene expression, a possible 15-ADON-specific pattern was observed for TRI12 encoding a trichothecene efflux pump. The expression levels of this pump reached a second peak at day 10 only in F. graminearum $\mathrm{PH}-1$ and $\mathrm{H}-11$, although the expression levels were lower than at days 2 or 4 .

In conclusion, the expression levels of most TRI genes essential for trichothecene production in both $F$. graminearum and $F$. asiaticum were strongly induced at early incubation times (i.e., 2 days after inoculation) before toxin biosynthesis. NIV chemotype-specific gene expression patterns were identified for two transcriptional regulator genes (TRI6 and TRI10), and the lower NIV production in F. asiaticum may be due to the lower expression levels of TRI genes in $F$. asiaticum relative to $F$. graminearum.

\section{Acknowledgements}

This study was carried out with the support of "Research Program for Agricultural Science \& Technology Development
(Project No. PJ008432)", National Academy of Agricultural Science, Rural Development Administration, Republic of Korea. HKK and SHY were supported by the Soonchunhyang University Research Fund.

\section{References}

Boddu, J., Cho, S., Kruger, W. M. and Muehlbauer, G. J. 2005. Transcriptome analysis of the barley-Fusarium graminearum interaction. Mol. Plant Microbe Interact. 19:407-417.

Brown, D. W., McCormick, S. P., Alexander, N. J., Proctor, R. H. and Desjardins, A. E. 2001. A genetic and biochemical approach to study trichothecene diversity in Fusarium sporotrichioides and Fusarium graminearum. Fungal Genet. Biol. 32:121-133.

Desjardins, A. E. 2006. Fusarium mycotoxins: chemistry, genetics, and biology. The American Phytopathological Society, St. Paul, Minnesota, USA. 260 pp.

Doohan, F. M., Weston, G., Rezanoor, H. N., Parry, D. W. and Nicholson, P. 1999. Development and use of a reverse transcription-PCR assay to study expression of Tri5 by Fusarium species in vitro and in planta. Appl. Environ. Microbiol. 65:3850-3854.

Dyer, R. B., Plattner, R. D., Kendra, D. F. and Brown, D. W. 2005. Fusarium graminearum TRI14 is required for high virulence and DON production on wheat but not for DON synthesis in vitro. J. Agric. Food Chem. 53:9281-9287.

Eriksen, G. S., Pettersson, H. and Lundh, T. 2004. Comparative cytotoxicity of deoxynivalenol, nivalenol, their acetylated derivatives and de-epoxy metabolites. Food Chem. Toxicol. 42:619-624.

Gale, L. R., Harrision, S. A., Ward, T. J., O’Donnell, K., Milus, E. A., Gale, S. W. and Kistler, H. C. 2011. Nivalenol-type populations of Fusarium graminearum and $F$. asiaticum are prevalent on wheat in southern Louisiana. Phytopathology 101:124-134.

Gardiner, D. M., Kazan, K. and Manners, J. M. 2009. Mutrient profiling reveals potent inducers of trichothecene biosynthesis in Fusarium graminearum. Fungal Genet. Biol. 46:604-613.

Hallen-Adams, H. E., Wenner, N., Kuldau, G. A. and Trail, F. 2011. Deoxynivalenol biosynthesis-related gene expression during wheat kernel colonization by Fusarium graminearum. Phytopathology 101:1091-1096.

Jiao, F., Kawakami, A. and Nakajima, T. 2008. Effects of different carbon sources on trichothecene production and Tri gene expression by Fusarium graminearum in liquid culture. FEMS Microbiol. Lett. 285:212-219.

Karugia, G. W., Suga, H., Gale, L. R., Nakajima, T., Ueda, A. and Hyakumachi, M. 2009. Population structure of Fusarium asiaticum from two Japanese regions and eastern China. $J$. Gen. Plant Pathol. 75:110-118.

Kim, H.-K., Cho, E. J., Lee, S., Lee, Y.-S. and Yun, S.-H. 2012. Functional analyses of individual mating-type transcripts at MAT loci in Fusarium graminearum and Fusarium asiaticum. 
FEMS Microbiol Lett. 337:89-96.

Kim, J. E., Han, K. H., Jin, J., Kim, H., Kim, J. C., Yun, S.-H. and Lee, Y.-W. 2005. Putative polyketide synthase and laccase genes for biosynthesis of aurofusarin in Gibberella zeae. Appl. Environ. Microbiol. 71:1701-1708.

Kim, H.-K. and Yun, S.-H. 2011. Evaluation of potential reference genes for quantitative RT-PCR analysis in Fusarium graminearum under different culture conditions. Plant Pathol. J. 27: 301-309.

Kimura, M., Tokai, T., O’Donnell, K., Ward, T. J., Fujimura, M., Hamamoto, H., Shibata, T. and Yamaguchi, I. 2003. The trichothecene biosynthesis gene cluster of Fusarium greminearum F15 contains a limited number of essential pathway genes and expressed non-essential genes. FEBS Lett. 539:105-110.

Kimura, M., Tokai, Takahashi-Ando, N., Ohsato, S. and Fujimura, M. 2007. Molecular and genetic studies of Fusarium trichothecene biosynthesis: pathways, genes, and evolution. Biosci. Biotechnol. Biochem. 71:2105-2123.

Lee, J., Chang, I. Y., Yun, S.-H., Leslie, J. F. and Lee, Y.-W. 2009. Genetic diversity and fitness of Fusarium graminearum populations from rice in Korea. Appl. Environ. Microbiol. 75:3289-3295.

Lee, T., Han, Y.-K., Kim, K.-H., Yun, S.-H. and Lee, Y.-W. 2002. Tri13 and Tri7 determine deoxynivalenol- and nivalenolproducing chemotypes of Gibberella zeae. Appl. Environ. Microbiol. 68:2148-2154.

Lee, T., Lee, S.-H., Lee, S.-H., Shin, J. Y., Yun, J.-C., Lee, Y.-W. and Ryu, J.-G. 2011. Occurrence of Fusarium mycotoxins in rice and its milling by-products in Korea. J. Food Prot. 74:1169-1174.

Lee, T., Oh, D.-W., Kim, H.-S., Lee, J., Kim, Y.-H., Yun, S.-H. and Lee, Y.-W. 2001. Identification of deoxynivalenol- and nivalenol-producing chemotypes of Gibberella zeae by using PCR. Appl. Environ. Microbiol. 67:2966-2972.

Leslie, J. F. and Summerell, B. A. 2006. The Fusarium laboratory manual. Blackwell Publishing Professional, Ames, Iowa, U.S.A. 388 pp.

Merhej, J., Boutigny, A. L., Pinson-Gadais, L., Richard-Forget, F. and Barreau, C. 2010. Acidic $\mathrm{pH}$ as a determinant of TRI gene expression and trichothecene B biosynthesis in Fusarium graminearum. Food Addit. Contam. 27:710-717.

Mule, G., Logrieco, A., Stea, G. and Bottalico, A. 1997. Clustering of trichothecene-producing strains determined from $28 \mathrm{~S}$ ribosomal DNA sequences. Appl. Environ. Microbiol. 63:18431846.

O'Donnell, K., Kistler, H. C., Tacke, B. K. and Casper, H. H. 2000. Gene genealogies reveal global phylogeographic structure and reproductive isolation among lineages of Fusarium graminearum, the fungus causing wheat scab. Proc. Natl. Acad. Sci. USA 97:7905-7910.

Pasquali, M., Giraud, F., Brochot, C., Cocco, E., Hoffmann, L. and Bohn, T. 2010. Genetic Fusarium chemotyping as a useful tool for predicting nivalenol contamination in winter wheat. Int. J. Food Microbiol. 137:246-253.

Peplow, A. W., Tag, A. G., Garifullina, G. F. and Beremand, M. N. 2003. Identification of new genes positively regulated by Tri10 and a regulatory network for trichothecene mycotoxin production. App. Environ. Microbiol. 69: 2731-2736.

Sambrook, J. and Russell, D. W. 2001. Molecular cloning: a laboratory manual. 3rd ed. Cold Spring Harbour Laboratory Press, Cold Spring Harbour, New York.

Sobrova, P., Adam, V., Vasatkova, A., Beklova, M., Zeman, L. and Kizek, R. 2010. Deoxynivalenol and its toxicity. Interdisciplinary Toxicol. 3:94-99.

Trail, F. and Common, R. 2000. Perithecial development by Gibberella zeae: a light microscopy study. Mycologia 92:130138.

Wang, J.-H., Ndoye, M., Zhang, J.-B., Li, H.-P. and Liao, Y.-C. 2011. Population structure and genetic diversity of the Fusarium graminearum species complex. Toxins 3:1020-1037.

Yli-Mattila, T. 2011. Detection of trichothecene-producing Fusarium species in cereals in Northern Europe and Asia. Agron. Res. 9:521-526.

Zhang, J.-B., Li, H.-P., Dang, F.-J., Qu, B., Xu, Y.-B., Zhao, C.S. and Liao, Y.-C. 2007. Determination of the trichothecene mycotoxin chemotypes and associated geographical distribution and phylogenetic species of the Fusarium graminearum clade from China. Mycol. Res. 111:967-975. 